\title{
Normative Hearing Threshold Levels in Koreans with Normal Tympanic Membranes and Estimated Prevalence of Hearing Loss
}

\author{
Hong Ju Park ${ }^{1}$ Myung Hoon Yoo ${ }^{1}$ Sun Young Baek ${ }^{2}$ Seon Woo Kim² $\cdot$ Yang-Sun Cho ${ }^{3}$ \\ ${ }^{1}$ Department of Otolaryngology, Asan Medical Center, University of Ulsan College of Medicine, Seoul; ${ }^{2}$ Biostatistics Team, Samsung Biomedical \\ Research Institute, Seoul; ${ }^{3}$ Department of Otorhinolaryngology-Head and Neck Surgery, Samsung Medical Center, Sungkyunkwan University
}

School of Medicine, Seoul, Korea

Objectives. We investigated the normative data on the hearing threshold levels of Koreans with normal tympanic membranes and the prevalence of hearing loss (HL) and nonserviceable hearing using the data from the Korea National Health and Nutrition Examination Surveys (KNHANES) during 2010-2012.

Methods. Data obtained from 16,673 participants $\geq 12$-year-of-age with normal tympanic membranes who completed audiometric testing. We defined $\mathrm{HL}$ as the pure tone average (PTA) $>25 \mathrm{~dB}$ hearing level at 500, 1,000, 2,000, and $3,000 \mathrm{~Hz}$ and non-serviceable hearing as PTA $>40 \mathrm{~dB}$ hearing level.

Results. The hearing levels at some frequencies $(0.5,3$, and $6 \mathrm{kHz})$ did not differ in between the 10 's and 20 's, but the hearing thresholds at all frequencies increased gradually from the 30's. The hearing thresholds were higher in men than in women at high frequencies $(3,4$, and $6 \mathrm{kHz}$ ) in the 30's and older. The prevalence of $\mathrm{HL}$ in either ear was $16.5 \%$ (estimates of 5.9 million), from $2.4 \%$ in the 10 's up to $75.4 \%$ in the 70 's and older. The prevalence of nonserviceable hearing in either ear was $6.8 \%$ (estimates of 2.5 million) and that of bilateral nonserviceable hearing was $2.5 \%$ (estimates of 0.9 million).

Conclusion. Hearing loss aggravated from the 30's at all frequencies and men showed poorer hearing levels than women at high frequencies. Hearing loss was a common condition and the prevalence of non-serviceable hearing in either ear, which needs hearing rehabilitation to help social communication, was $6.8 \%$. Normative pure tone thresholds at each frequency can be used as referent values when counseling patients complaining of hearing loss.

Keywords. Hearing Loss; Normative Data; Pure Tone; Audiometry; Thresholds

\section{INTRODUCTION}

Hearing loss (HL) is well-known to be highly prevalent, and the prevalence is expected to rise significantly with progression of the aging process. Hearing loss can impair meaningful commu-

\footnotetext{
- Received January 5, 2016

Revised March 16, 2016

Accepted June 17, 2016

- Corresponding author: Yang-Sun Cho

Department of Otorhinolaryngology-Head and Neck Surgery, Samsung

Medical Center, Sungkyunkwan University School of Medicine, 81

Irwon-ro, Gangnam-gu, Seoul 06351, Korea

Tel: +82-2-3410-3579, Fax: +82-2-3410-3879

E-mail: yangsun.cho@gmail.com
}

nication and social connectivity and it is significantly related with depression regardless of age and socioeconomic state [1]. Though there have been a few reports about audiologic data and age-related hearing loss in Koreans [2-4], there has been no report on normative audiologic data based on the distribution in Korean population with normal tympanic membranes. Level of hearing may be variable according to the demographic characteristics such as gender, ethnicity, and age [5,6]. Therefore, comparison with a demographically-matched reference group is required to determine whether a person's hearing is normal. This information can alert clinicians or patients to the need for additional diagnostic information in cases where the hearing is out of normal range. Furthermore, the representative estimates of

Copyright @ 2017 by Korean Society of Otorhinolaryngology-Head and Neck Surgery.

This is an open-access article distributed under the terms of the Creative Commons Attribution Non-Commercial License (http://creativecommons.org/licenses/by-nc/4.0)

which permits unrestricted non-commercial use, distribution, and reproduction in any medium, provided the original work is properly cited. 
the population with hearing loss may support the proper distribution of public health resources for hearing rehabilitation.

The present study was undertaken to report the national audiologic reference data and the prevalence of age-related hearing loss based on the audiometric testing in Koreans with normal tympanic membranes from the Korea National Health and Nutrition Examination Surveys (KNHANES) during 20102012.

\section{MATERIALS AND METHODS}

\section{Study population and data collection}

The KNHANES started in 1998 to examine the general health and nutrition status of populations in South Korea. The KNH ANES is an ongoing cross-sectional survey of the civilian noninstitutionalized population of South Korea. A field survey team that included an otolaryngologist, an ophthalmologist and nurse examiners for health assessment moved with a mobile examination unit and performed interviews and physical examinations. Participants underwent a basic health assessment and otolaryngologic examination. Close history of otologic symptoms was surveyed and physical examination of the tympanic membrane, and the evaluation of hearing was conducted in participants of appropriate ages. Every year, individuals in about 4,600 households are selected from a panel to represent the population by using the multistage clustered and stratified random sampling method that is based on the National Census Data. The participation rate of selected households in the past several cycles of KNHANES was high, ranging from $76 \%$ to $82 \%$. Although individual participants were not equally representative of the Korean population, this survey provides representative estimates of the noninstitutionalized Korean civilian population by using the power of sample weight. A sample weight was assigned to each sample individual through three steps, calculating the base weight which is the inverse of probability for an individual to be selected, adjusting for nonresponse, and poststratification adjustment to match census population control.

The study population was 16,673 participants with normal tympanic membranes over 12-year-of-age who completed audiometric testing during 2010-2012. Any participants with abnormal tympanic membranes $(\mathrm{n}=3,767),<12$ years of age $(\mathrm{n}=$ $2,008)$ or insufficient data $(n=1,173)$ were excluded. They rep-

\section{H I G H L I G H T S}

- Hearing is aggravated from the 30's at all frequencies.

- The prevalence of non-serviceable hearing in either ear was $6.8 \%$ (2.5 million) in Korea.

- Normative hearing thresholds can be used as a reference when counseling on hearing loss. resent the 35,882,248 individuals in South Korea $\geq 12$ years of age. Written informed consents were obtained from all the participants prior to the survey and approval for this research was obtained from the Institutional Review Board of the Samsung Medical Center (IRB no. 2013-02-031).

\section{Otologic examination and audiometric measurement}

An ear examination was conducted with a 4-mm $0^{\circ}$-angled rigid endoscope attached to a charge coupled device (CCD) camera for all participants to find tympanic membrane perforation or cholesteatoma, retraction pocket, otitis media with effusion or abnormal external auditory canal.

The pure tone air-conduction threshold was measured in a sound-proof booth using an automatic audiometer (GSI SA203, Entomed Diagnostics AB, Lena Nodin, Sweden). Six hearing thresholds were measured in each ear by pure tone audiometry using an ascending/descending technique in $5-\mathrm{dB}$ steps at the frequencies of $0.5,1,2,3,4$, and $6 \mathrm{kHz}$. The order of sound frequency was assigned in a random way. Hearing loss was defined as $>25 \mathrm{~dB}$ HL in average of air-conduction hearing thresholds at $0.5,1,2$, and $3 \mathrm{kHz}$ and nonserviceable hearing was defined as $>40 \mathrm{~dB} \mathrm{HL}$ in average of air-conduction hearing thresholds at $0.5,1,2$, and $3 \mathrm{kHz}$. The data were divided in seven age groups $(12-19 ; 20-29 ; 30-39 ; 40-49 ; 50-59 ; 60-69 ; 70$ and older) and thresholds were presented separately for age and gender groups. Bone-conduction thresholds were not measured in this survey.

For normative hearing threshold levels, those from the better ears were presented in centiles at each specific frequency from 250 to $6,000 \mathrm{~Hz}$. The sample was divided into seven age groups (12-19; 20-29; 30-39; 40-49; 50-59; 60-69; 70 and older) and the hearing thresholds were presented separately for gender and age groups. In order for the hearing thresholds to be representative for the population, they were weighted on age and gender according to the participation rate in the study.

\section{Statistical analysis}

All statistical analysis was performed taking account of weights from the complex sampling design according to the guideline for analysis of KNHAENS data from Korea Centers for Disease Control and Prevention. The sampling weights were adjusted so that the total sample will represent the population for the 3-year period (2010-2012), and weight for each individual from each year divided by 3 was used in the analysis. The prevalence for hearing loss and $95 \%$ confidence interval (CI) were estimated. We analyzed the linear trend of prevalence with age using logistic regression. Two sample $t$-test was used to test the difference of hearing thresholds between gender at each frequency. Tukey's test for multiple comparisons was used to test the difference of hearing thresholds among age groups at each frequency. Bonferroni correction was applied to the $P$-value due to multiple testing or subgroup analysis. $P$-values were two-sided, and $P<0.05$ 
was considered to be statistically significant. Statistical analysis was executed using SAS ver. 9.3 (SAS Inc., Cary, NC, USA).

\section{RESULTS}

\section{The hearing threshold levels by age and gender}

The hearing threshold results for each gender and age group are presented in Table 1. The mean thresholds for each frequency as a function of age in men and women are shown in Fig. 1. Overall, the hearing levels at some frequencies $(0.5,3$, and $6 \mathrm{kHz})$ did not differ in between the 10's and 20's, but the hearing thresholds at all frequencies increased gradually from the 30's. In detail, there was no significant difference in the 10's and 20's at $0.5,3$, and $6 \mathrm{kHz}$ and hearing thresholds increased with ages beyond the 20's at those frequencies $(P<0.001$, Tukey's test for multiple comparisons). At other frequencies (1, 2, and $4 \mathrm{kHz})$, hearing thresholds increased throughout the ages $(P<0.001$, Tukey's test for multiple comparisons). More specifically (Fig. 2), women did not show a significant difference in hearing levels in between the 10's and 20's in all frequencies and hearing thresh- olds deteriorated with ages beyond the 20's at all frequencies $(P<0.001)$. In men, there was no significant difference in hearing levels in between the 10's and 20's in all frequencies except $4 \mathrm{kHz}$ and hearing thresholds increased with ages beyond the 20's at all frequencies $(P<0.001)$.

When comparing the hearing levels in men and women, the hearing thresholds were higher in men than in women at high frequencies $(3,4$, and $6 \mathrm{kHz})$ in the 30's and older (Table 2). In the 10 's, there was no significant difference of hearing levels between men and women at all frequencies except $6 \mathrm{kHz}$. In the 20 's, there was no significant difference of hearing levels between men and women at all frequencies. In the 30's and older, the hearing levels were higher in men than in women at high frequencies $(3,4$, and $6 \mathrm{kHz})$. Overall, men showed higher hearing levels at high frequencies $(3,4$, and $6 \mathrm{kHz})$ and lower hearing levels at low frequencies $(0.5$ and $1 \mathrm{kHz})$ than women $(P<0.001$, by two sample $t$-test).

\section{The prevalence of hearing loss according to the laterality and severity}

Among the 16,673 participants $\geq 12$-year-of-age, the prevalence
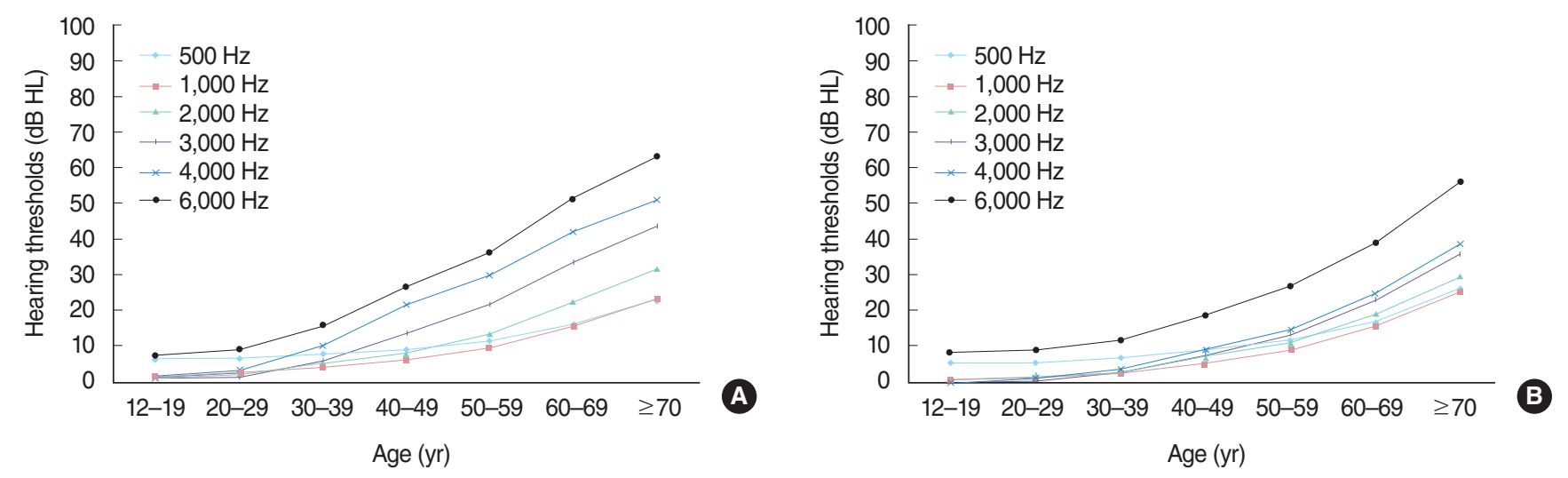

Fig. 1. Mean hearing threshold levels at each frequency in Korean men (A) and women (B).
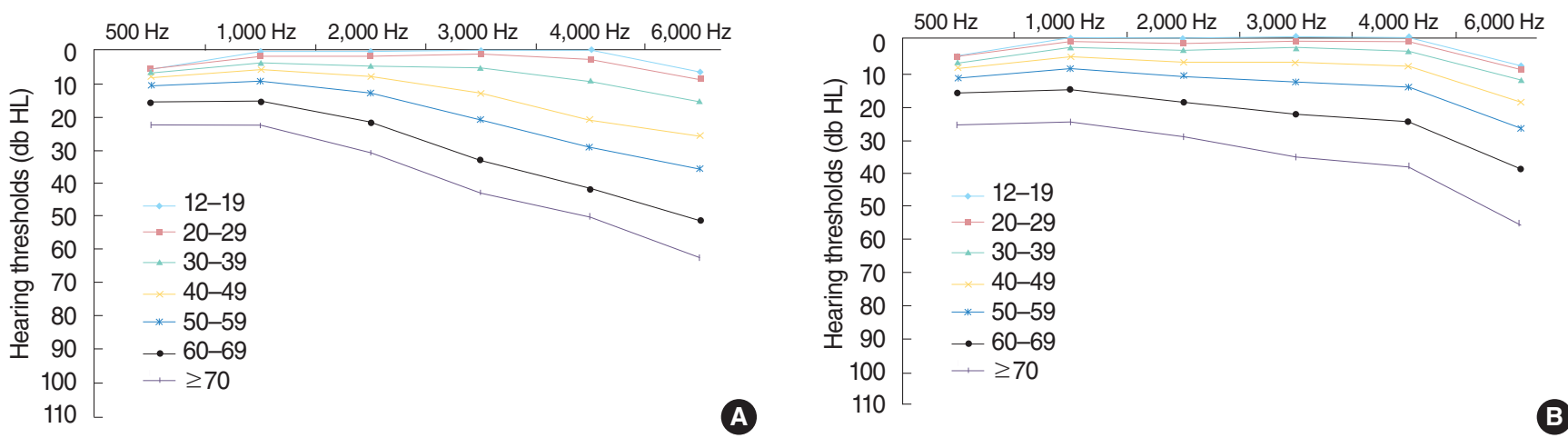

Fig. 2. Changes of mean hearing levels with ages in Korean men $(A)$ and women $(B)$. In men, there was no significant difference in hearing levels in between the 10's and 20's in all frequencies except $4 \mathrm{kHz}$ and hearing thresholds increased with ages beyond the 20's at all frequencies $(P<0.001)$. Women showed no significant difference in hearing levels in between the 10's and 20's in all frequencies and hearing thresholds increased with ages beyond the 20's at all frequencies $(P<0.001)$. 


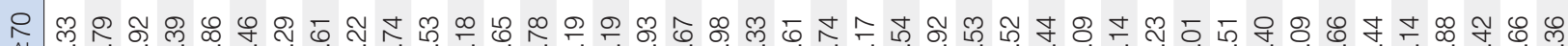

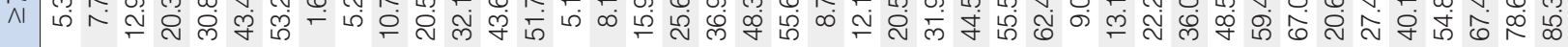

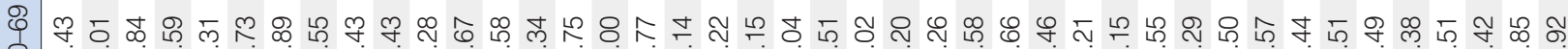
ठ

유 ద

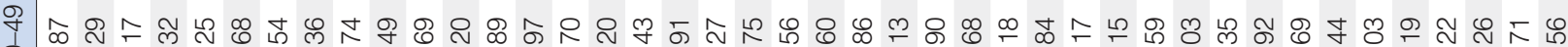
们

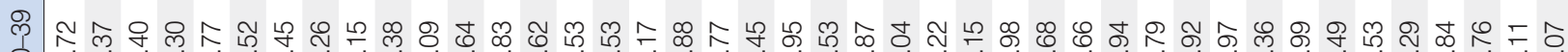

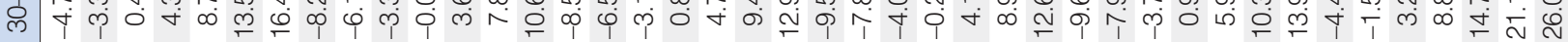

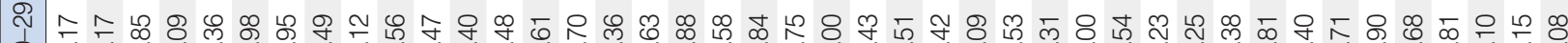

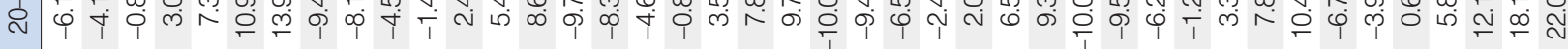

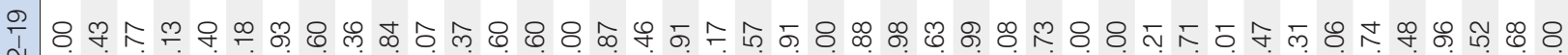
$\stackrel{\sim}{\sim}$ 枿

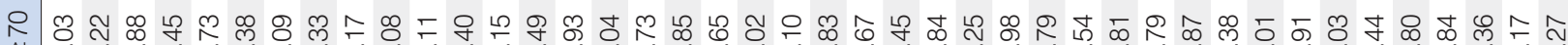
^

\& ৫ T

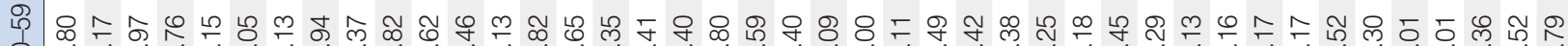
চ

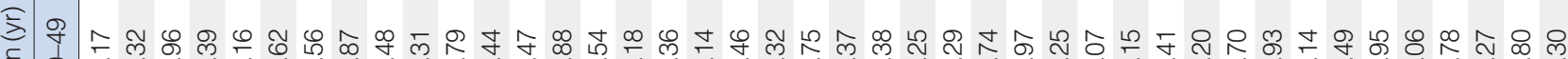
மு

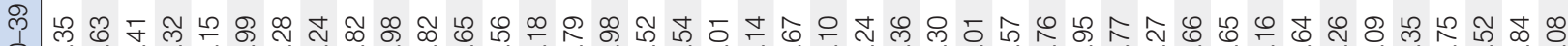
ल L

令 ๙

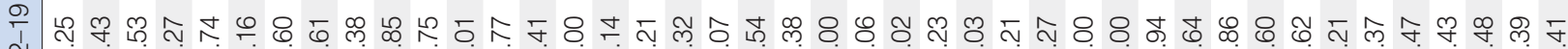

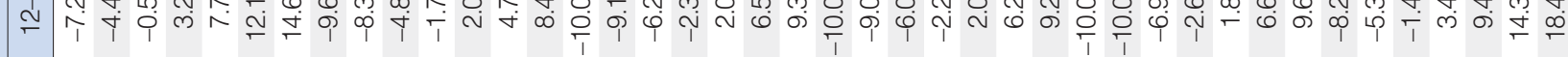

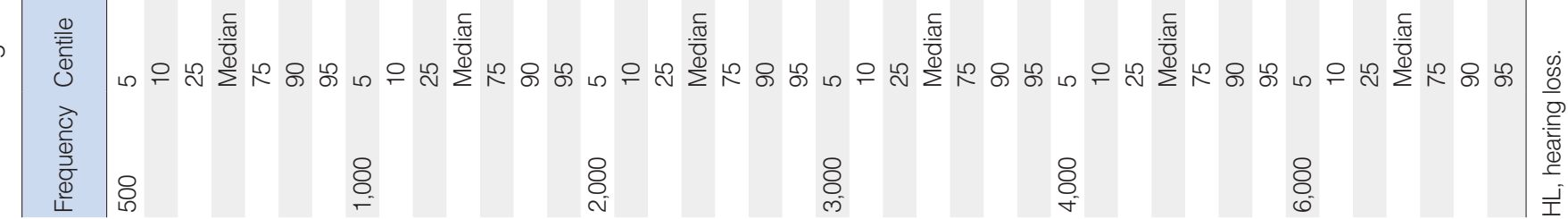



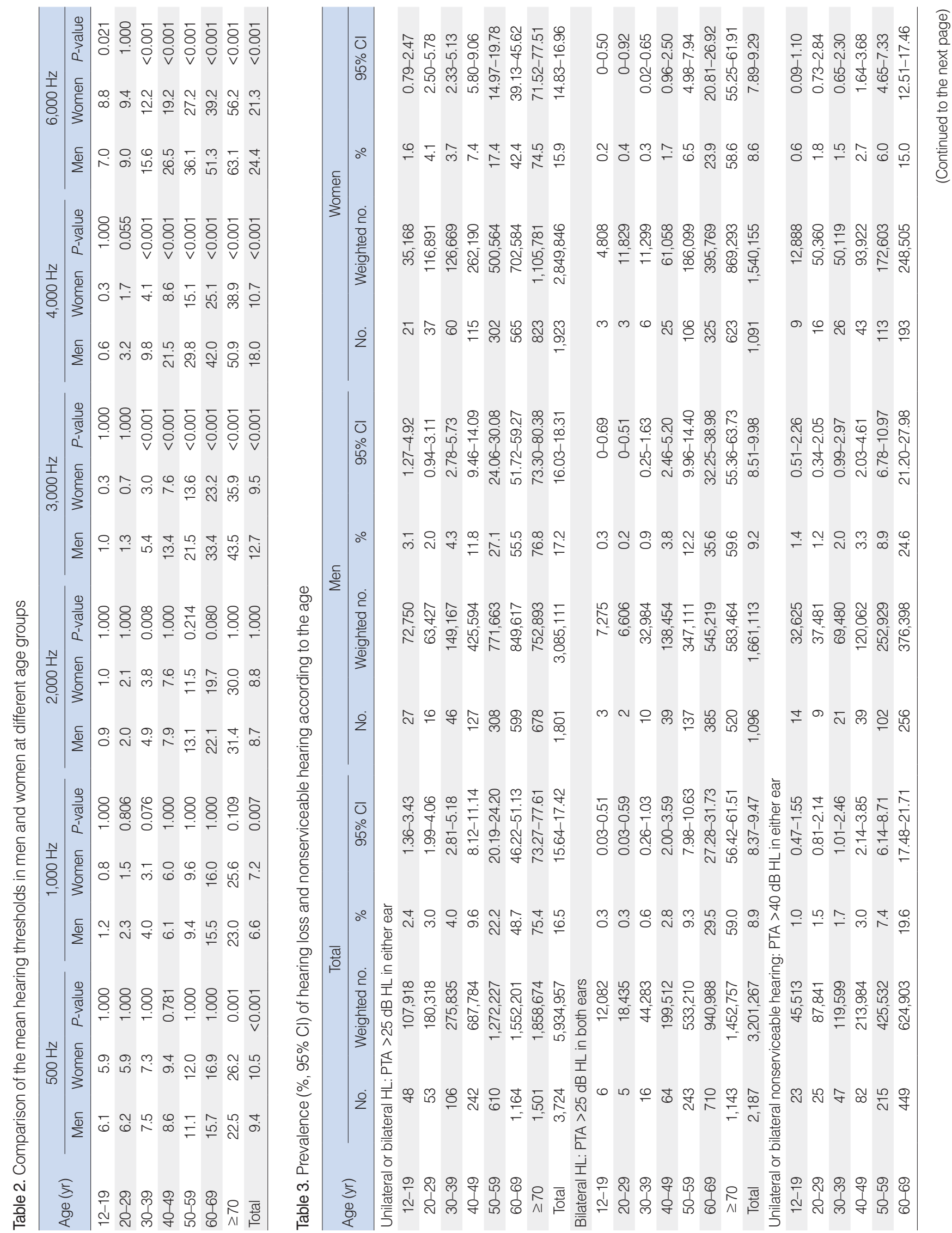


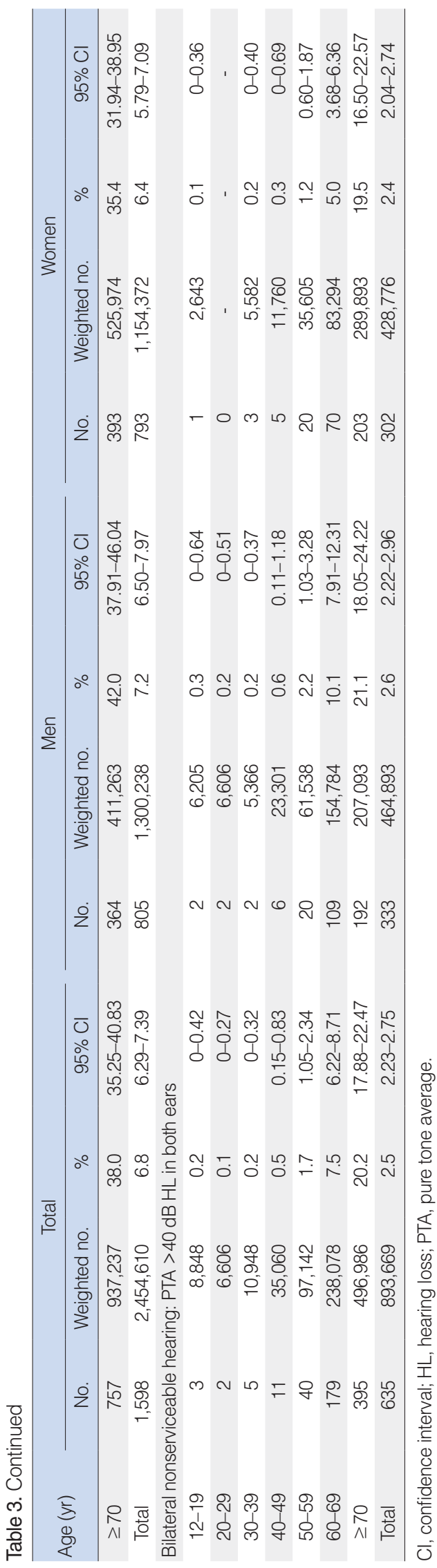

of hearing loss (pure tone average [PTA] $>25 \mathrm{~dB}$ hearing level) in either ear of participants was $16.5 \%$ (estimates of 5.9 million) (Table 3). In the youngest age group (12-19 years), 2.4\% exhibited hearing loss, and the prevalence rate increased with age up to $75.4 \%$ in oldest group (70 years and older). The prevalence rate showed an age-related increase at all frequencies $(P<0.001$ by logistic regression analysis). The prevalence of bilateral $\mathrm{HL}$ was $8.9 \%$ (estimates of 3.2 million). In the youngest age group (12-19 years), $0.3 \%$ exhibited bilateral HL, and the prevalence rate increased with age up to $59.0 \%$ in oldest group (70 years and older).

The prevalence of nonserviceable hearing (PTA $>40 \mathrm{~dB}$ hearing level) in either ear of participants was $6.8 \%$ (estimates of 2.5 million). In the youngest age group (12-19 years), 1.0\% exhibited hearing loss of $>40 \mathrm{~dB}$, and the prevalence rate increased with age up to $38.0 \%$ in oldest group (70 years and older). The prevalence rate showed an age-related increase at all frequencies ( $P<0.001$ by logistic regression analysis). The prevalence of bilateral nonserviceable hearing was $2.5 \%$ (estimates of 0.9 million). In the youngest age group (12-19 years), $0.2 \%$ exhibited bilateral hearing loss of $>40 \mathrm{~dB}$, and the prevalence rate increased with age up to $20.2 \%$ in oldest group $(P<0.001$ by logistic regression analysis).

\section{DISCUSSION}

To our knowledge, there has been no report about normative values of hearing thresholds in Asian populations through a national survey, though there have been several reports from regional survey [7-11]. Table 1 displays the statistical distribution of thresholds of the Korean men and women by age. The hearing threshold values presented in this survey provide the expected values of hearing thresholds for Koreans with normal tympanic membranes. This information may be useful for clinical counseling with younger individuals presenting for audiological assessment.

The hearing thresholds in 10's and 20's (audiological reference population) obtained in this survey are around 0-6 dB HL from $500 \mathrm{~Hz}$ to $4,000 \mathrm{~Hz}$. Theoretically, the expected value, according to the international standard, is supposed to be $0 \mathrm{~dB} \mathrm{HL}$ (ISO 7029). There are several factors related to the testing procedures. The ambient noise in the test area and the use of a 5-dB step size in test signal presentation compared to smaller $2-\mathrm{dB}$ steps as used in other studies may contribute to this discrepancy [12]. However, taking into account the negligible difference $(\leq 1$ $\mathrm{dB}$ ) between the thresholds in this study and $0 \mathrm{~dB}$ HL especially at 1,2 , and $3 \mathrm{kHz}$ (Table 1), our audiologic data may represent the reference data base for the distribution of normal Korean population. It was reported that the median hearing threshold levels of any group of 18-20 years olds, are not zero, but on the order of $+5 \mathrm{~dB}$ for most frequencies of $0.25-8 \mathrm{kHz}$ in audio- 
metric surveys [13].

There have been several reports about the prevalence of hearing loss. The present prevalence estimate of $16.5 \%$ (based on participants aged 12 years or older) is lower than those $(20.3 \%-$ $26.1 \%$ ) derived from different countries including United Kingdom, Australia, and United States [7,14-16]. This difference can be explained partially by the fact that we excluded any participants with abnormal tympanic membrane in this study. A recent KHANES study of the same period showed that the prevalence of hearing loss in Korean population was $22.7 \%$, when tympanic membrane abnormalities were included [17]. Similarly, recent work has demonstrated that $20.3 \%$ of Americans 12 years and older have hearing loss (NHANES 2001-2008) [16]. Though the prevalence rates from national estimates may be variable according to the differences in age groups and ethnic distributions of the surveyed population, nation-wide epidemiological studies that are conducted by government organizations can provide powerful data for investigating the national prevalence of disease conditions. The representative estimates of the Korean civilian population suffering from hearing loss by using the power of sample weight may facilitate the proper and predictable allocation of public health resources aimed at hearing rehabilitation.

Our data clearly show that the prevalence of hearing loss increased with age. Age-related hearing loss may affect neural fibers, stria vascularis, and inner and outer hair cells causing progressive hearing impairment $[18,19]$. In the youngest age group (12-18 years), 2.4\% exhibited hearing loss in either ear, and the prevalence rate increased with age up to $75.4 \%$ in oldest group (70 years and older), which is similar to previous reports from other countries [7,14-16]. An estimate of 3.2 million (a prevalence of $8.9 \%$ ) had bilateral hearing loss in South Korea and 2.5 million (a prevalence of $6.8 \%$ ) had nonserviceable hearing. There is a strong relationship between hearing loss in the elderly and psychosocial status, which can lead to social isolation, cognitive decline and the loss of independence [20,21]. The most common audiologic rehabilitation approach to address adult-onset hearing impairment is the provision of hearing aids, which is well-known to improve individual quality of life [22,23]. Due to the increase in life expectancy, hearing rehabilitation should be considered for an ageing population with hearing loss.

There are several limitations to this study. We tried to exclude conductive hearing losses by excluding any participants with abnormal tympanic membrane. As only air-conduction threshold was evaluated and bone-conduction pure tone testing was not performed in this survey, conductive hearing losses could not be entirely excluded. Also, we did not subcategorized hearing losses by specific etiologies. Particularly, the history of exposure to noise was not an exclusion criterion, given that noise is well known to induce hearing loss [24].

In conclusions, hearing loss aggravated from the 30's at all frequencies and men showed higher hearing thresholds than women at high frequencies. Hearing loss was a common condition and the prevalence of nonserviceable hearing in either ear, which needs hearing rehabilitation to help social communication, was $6.8 \%$. Normative pure tone thresholds at each frequency can be used as referent values when counseling patients complaining of hearing loss.

\section{CONFLICT OF INTEREST}

No potential conflict of interest relevant to this article was reported.

\section{ACKNOWLEDGMENTS}

We appreciate Korean Society of Otorhinolaryngology-Head and Neck Surgery and members of the Division of Chronic Disease Surveillance in Korea Centers for Disease Control \& Prevention for participating in this survey and the dedicated work they provided.

\section{REFERENCES}

1. Herbst KG, Humphrey C. Hearing impairment and mental state in the elderly living at home. Br Med J. 1980 Oct;281(6245):903-5.

2. Kim S, Lim EJ, Kim HS, Park JH, Jarng SS, Lee SH. Sex differences in a cross sectional study of age-related hearing loss in Korean. Clin Exp Otorhinolaryngol. 2010 Mar;3(1):27-31.

3. Cho YS, Choi SH, Park KH, Park HJ, Kim JW, Moon IJ, et al. Prevalence of otolaryngologic diseases in South Korea: data from the Korea national health and nutrition examination survey 2008. Clin Exp Otorhinolaryngol. 2010 Dec;3(4):183-93.

4. Kim HN, Kim SG, Lee HK, Ohrr H, Moon SK, Chi J, et al. Incidence of presbycusis of Korean populations in Seoul, Kyunggi and Kangwon provinces. J Korean Med Sci. 2000 Oct;15(5):580-4.

5. Cooper JC Jr. Health and Nutrition Examination Survey of 197175: part I. ear and race effects in hearing. J Am Acad Audiol. 1994 Jan;5(1):30-6.

6. Helzner EP, Cauley JA, Pratt SR, Wisniewski SR, Zmuda JM, Talbott $\mathrm{EO}$, et al. Race and sex differences in age-related hearing loss: the Health, Aging and Body Composition Study. J Am Geriatr Soc. 2005 Dec;53(12):2119-27.

7. Borchgrevink HM, Tambs K, Hoffman HJ.The Nord-Trondelag Norway Audiometric Survey 1996-98: unscreened thresholds and prevalence of hearing impairment for adults $>20$ years. Noise Health. 2005 Jul-Sep;7(28):1-15.

8. Flamme GA, Deiters K, NeedhamT. Distributions of pure tone hearing threshold levels among adolescents and adults in the United States by gender, ethnicity, and age: results from the US National Health and Nutrition Examination Survey. Int J Audiol. 2011 Mar; 50 Suppl 1:S11-20.

9. Rodriguez Valiente A, Roldan Fidalgo A, Garcia Berrocal JR, Ramirez Camacho R. Hearing threshold levels for an otologically screened population in Spain. Int J Audiol. 2015 Aug;54(8):499-506.

10. Lin CY, Yang YC, Guo YL, Wu CH, Chang CJ, Wu JL. Prevalence of hearing impairment in an adult population in Southern Taiwan. Int J Audiol. 2007 Dec;46(12):732-7. 
11. Molvaer OI, Vardal L, Gundersen T, Halmrast T. Hearing acuity in a Norwegian standard population. Scand Audiol. 1983;12(4):229-36.

12. Williams W, Carter L, Seeto M. Hearing threshold levels for a population of 11 to 35 year old Australian females and males. Int J Audiol. 2014 May;53(5):289-93.

13. Borchgrevink HM. Does health promotion work in relation to noise? Noise Health. 2003 Jan-Mar;5(18):25-30.

14. Davis AC. The prevalence of hearing impairment and reported hearing disability among adults in Great Britain. Int J Epidemiol. 1989 Dec;18(4):911-7.

15. Wilson DH, Walsh PG, Sanchez L, Davis AC, Taylor AW, Tucker G, et al. The epidemiology of hearing impairment in an Australian adult population. Int J Epidemiol. 1999 Apr;28(2):247-52.

16. Lin FR, Niparko JK, Ferrucci L. Hearing loss prevalence in the United States. Arch Intern Med. 2011 Nov;171(20):1851-2.

17. Jun HJ, Hwang SY, Lee SH, Lee JE, Song JJ, Chae S. The prevalence of hearing loss in South Korea: data from a population-based study. Laryngoscope. 2015 Mar;125(3):690-4.

18. Schuknecht HF, Gacek MR. Cochlear pathology in presbycusis. Ann Otol Rhinol Laryngol. 1993 Jan;102(1 Pt 2):1-16.

19. Mills DM, Schmiedt RA. Metabolic presbycusis: differential changes in auditory brainstem and otoacoustic emission responses with chronic furosemide application in the gerbil. J Assoc Res Otolaryngol. 2004 Mar;5(1):1-10.

20. Gussekloo J, de Bont LE, von Faber M, Eekhof JA, de Laat JA, Hulshof $\mathrm{JH}$, et al. Auditory rehabilitation of older people from the general population: the Leiden 85-plus study. Br J Gen Pract. 2003 Jul;53(492):536-40.

21. Yueh B, Collins MP, Souza PE, Boyko EJ, Loovis CF, Heagerty PJ, et al. Long-term effectiveness of screening for hearing loss: the screening for auditory impairment: which hearing assessment test (SAIWHAT) randomized trial. J Am Geriatr Soc. 2010 Mar;58(3):42734.

22. Boothroyd A. Adult aural rehabilitation: what is it and does it work? Trends Amplif. 2007 Jun;11(2):63-71.

23. Chisolm TH, Johnson CE, Danhauer JL, Portz LJ, Abrams HB, Lesner S, et al. A systematic review of health-related quality of life and hearing aids: final report of the American Academy of Audiology Task Force On the Health-Related Quality of Life Benefits of Amplification in Adults. J Am Acad Audiol. 2007 Feb;18(2):151-83.

24. AgrawalY, Platz EA, Niparko JK. Prevalence of hearing loss and differences by demographic characteristics among US adults: data from the National Health and Nutrition Examination Survey, 1999-2004. Arch Intern Med. 2008 Jul;168(14):1522-30. 